\title{
Ultimatum Game Model Based on Facial Trait Inference
}

\author{
Yujia Wu \\ Beijing University of Posts and Telecommunications, Beijing 100876, China \\ wuyujia@bupt.edu.cn
}

\begin{abstract}
The existence of a beauty premium in the labor market suggests that appearance can exert effect in the real world. We explored facial trait inference-especially focused on facial attractiveness and facial trustworthiness-in an Ultimatum Game experiment, which is commonly used to examine economic decision making [1]. Based on the traditional FS model, which clearly described the function of ultimatum game, we modified and made improvements of it and constructed a model that was appropriate for our research. These findings point to important effects of facial trait inferences on decision and provide initial insight into the psychological processes regarding to the decision behavior.
\end{abstract}

Keywords: Ultimatum game; Trait inference; FS model.

\section{Introduction}

Facial imagery almost exists in every media in our daily life, like television, printed advertising, webpage etc. A proverb says 'judge the book by its cover', which means people often form first impressions about others based on their facial appearance and subsequently use these judgments or impressions to guide their own behavior. The simple reality is that there are a tremendous variety of circumstances and contexts where automatic processing of facial stimuli may influence decision process. Prior research has found that people frequently rely on facial appearance to draw trait inferences about others. The ability to infer social traits is a crucial component of social functioning and development and is an efficient and effective strategy to make interpersonal decisions during social interactions especially when other information is lacking. Trustworthiness and attractiveness judgments in a face convey particularly pivotal social information and they are the two commonly social decisions made in a face. Much of the research in this area has studied how these inferences affect people's decision and behavior. Andreoni and Petrie (2008), for example, demonstrated that in a public goods game, participants cooperated more with attractive than less attractive partners and expected that attractive partners would be cooperative [2]. Consequently, attractive partners earned more than less attractive partners. In a trust game, Wout et al. (2008) explored the influence of facial trustworthiness on decision. The result showed that compared to untrustworthy-looking individuals, people would invest more money in trustworthy-looking individuals [3].

The ultimatum game is commonly used to examine economic decision-making [1]. Currently, the most widely adopted model to describe ultimatum game is FS model put forward by Fehr and Schmidt (2001) [4]. The present research contributes to this area by modifying the FS model to include the facial trustworthiness and attractiveness and demonstrating their effects on economic decision. The findings shed light on how the facial attractiveness and trustworthiness affect decision process. The research has theoretical implications for researchers to have a deep understanding about how people automatically code and respond to facial stimuli. It's of great importance for the researchers to know the influence processing of trait inferences on decision.

\section{Research Background}

In the ultimatum game, a proposer is given a sum of money to divide between himself or herself and a responder. The responder then has the option to accept or reject the allocation. If the responder accepts, both players receive the amount that has been allocated to them; if the responder rejects, both players get nothing. In order to determine whether the distribution of interests was fair, FS model proposed that people would make comparisons between interests of others and themselves. When their own interests are lower than others, it will create envy negative utility; when their own interests 
are higher than others, it will create sympathy negative utility. Total utility is equal to the sum of the direct utility brought by interests, envy negative utility and sympathy negative utility. The total utility function described as follows:

$$
u_{i}=x_{i} \quad \frac{i}{n 1_{j i}} \max \left(x_{j} x_{i}, 0\right) \frac{i}{n 1_{j i}} \max \left(x_{i} x_{j}, 0\right)
$$

In the above model, $i$ stands for a particular person, $\mathrm{n}$ stands for the total number in reference group; $j \neq i$ stands for people's comparison between their own interests and another people in the reference group; $\alpha_{i}$ stands for the intensity of envy of $i$ when his/her is lower than the other; $\beta i$ stands for the intensity of sympathy when his/her is higher than the other; the first item in the utility function $x_{i}$ stands for direct utility of $i$ brought by interests; the second item stands for total envy negative utility when one is compared with all the others in the group; the third item stands for total sympathy negative utility when one is compared with all the others in the group. Regularly $\alpha_{i}>\beta i$ and $1>\beta i \geqslant 0$. The former means the envy negative utility is greater than sympathy negative utility when both are caused by equal interests difference. The latter means although it will create sympathy negative utility when own interests is higher than the other, people still hope themselves have relatively more benefits than the other.

In the ultimatum game, there are only two participants: the proposer and the responder. Formula 1 can then be simplified as follows:

$$
u_{i}=x_{i}-\alpha_{i} \max \left(x_{j}-x_{i}, 0\right)-\beta i \max \left(x_{i}-x_{j}, 0\right)
$$

Previous researches revealed that the average of allocation portion proposed by proposer was between $40 \%$ and $50 \%$. The amount lower than $40 \%$ of total is often rejected by responder [5]. In this research, we only considered condition that the allocation for proposer is no less than for responder and focus on the utility function for responder. Formula 2 can then be simplified as follows:

$$
u_{r}=x_{r}-\alpha_{r}\left(x_{p}-x_{r}\right)
$$

The subscript $\mathrm{r}$ stands for responder, $\mathrm{p}$ stands for proposer, ${ }_{r}$ stands for the coefficient of envy of responder, $x_{r}$ stands for the allocation for responder, $x_{p}$ stands for the allocation for proposer. We hypothesis the total money is 1 unit and y means the allocation for responder, which should meet the following conditions:

$$
x_{p}+x_{r}=1 ; y=x_{r} ; 0 \leq y \leq \frac{1}{2}
$$

We took formula 4 into formula 3 and we got formula 5:

$$
u_{r}=\left(2 \alpha_{r}+1\right) y-\alpha_{r}
$$

\section{Research Hypothesis and Model}

\subsection{Research Hypothesis.}

H1: The facial attractiveness of proposer may have an effect on the envy effect of responder indirectly, which were non-linear changes.

$\mathrm{H} 2$ : The facial trustworthiness of proposer may have an effect on total utility of responder directly, which also were non-linear changes.

H3: The responder's evaluation of facial attractiveness of proposer may be influenced by the subjective evaluation criterion of facial attractiveness.

H4: The responder's evaluation of facial trustworthiness of proposer may be influenced by the subjective evaluation criterion of facial trustworthiness.

Description of the above hypothesis:

1) Previous research found the unfair allocation would bring responder negative emotional experience [6]. Meanwhile facial attractiveness would bring people positive emotional experience [7]. 
Therefore we hypothesis facial attractiveness would influence the envy utility directly and total utility indirectly.

2) Trust is an important factor in decision-making. Meanwhile facial trustworthiness provides important information for people's trust toward others [8]. So we hypothesis facial trustworthiness of proposer would influence the total utility of responder directly.

3) Even in the face of the same people, each individual has his/her own evaluation criterion. So we cannot ignore the influence of the individual subjective evaluation criterion when we consider the total utility. When faced the same face, for example, one may rate it 7 whose average evaluation criterion for attractiveness is 3, while another one may rate it 6 whose average evaluation criterion for attractiveness is 4 . Consequently the utilities are not the same.

\subsection{Research Model.}

Based on the above hypothesis, a new research model were built:

$$
\begin{aligned}
& u_{r}=\left(2 \alpha_{r}+1\right) y-\alpha_{r}+\beta_{r}\left(N_{\mathrm{p}}-v_{r}\right) \\
& \left.r=\left[\ln \frac{M_{p} r}{\max \left(M_{p} r\right.}\right)^{+1}\right], \quad \geq 0, M_{p} \geq r \text {; } \\
& \left.r=\left[\ln \frac{M_{p} r}{\max \left(M_{p} r\right.}\right)^{+1}\right], \geq 0, M_{p}<r
\end{aligned}
$$

Decision criterion: responder accept the allocation when $u_{r} \quad 0$; on the contrast, responder reject the allocation when $u_{r}<0$.

The interpretation of the above formula:

y: Allocation for responder;

$\alpha_{r}$ : Envy function, which meant the value of envy when responder's interests was lower than the proposer. It's absolute value of negative effect.

$\beta_{r}$ : Trustworthiness coefficients, which meant the effect of perceived trustworthiness of proposer on responder. It's absolute value of negative or positive effect.

$M_{p}$ : Responder's evaluation on proposer's facial attractiveness.

$N_{p}$ : Responder's evaluation on proposer's facial trustworthiness.

$\mu_{r}$ : Responder's average subjective evaluation on facial attractiveness.

$v_{r}$ : Responder's average subjective evaluation on facial trustworthiness.

$\eta$ : Envy coefficient of responder. The higher of envy coefficient, the more sensitive to envy.

$u_{r}$ : Total utility of responder when the allocation was $\mathrm{y}$.

(Note: the purpose of '-'in $r$ was to ensure the value was negative when ${ }^{M}{ }_{p}{ }_{r}$.

\section{Numerical Example}

We hypothesized that people make subjective evaluations of faces on Likert 7-scale and the average evaluations are around 4. We then adopted the Round function in Mtalab to generate four groups of random numbers and each group contained 100 numbers, showed as follows:

$$
\begin{aligned}
& M_{p}=1+\operatorname{round}(6 * \operatorname{rand}(100,1)) ; N_{p}=1+\operatorname{round}(6 * \operatorname{rand}(100,1)) ; \\
& u_{r}=4+\operatorname{round}(1 * \operatorname{rand}(100,1)) ; v_{r}=4+\operatorname{round}(1 * \operatorname{rand}(100,1))
\end{aligned}
$$


We then hypothesized $r_{r}=0.25,=0.3, \mathrm{y}=0.3$. According to formula 6 , we got 100 values of $u_{r}$. The result revealed that with the increase of $\mathrm{M}_{\mathrm{p}}$ and $\mathrm{N}_{\mathrm{p}}$, the total utility function increased from negative to positive, which meant that people would accept the unfair allocation with the increase of facial attractiveness and trustworthiness of proposer gradually.

\section{Summary}

This research focused on the ultimatum game model which contained two subjects. Through modifying the traditional FS model, the research construct an ultimatum game model based on facial trait inferences---facial attractiveness and facial trustworthiness. By generating random numbers, we analyzed the effect tendency of facial trait inferences on unfair decision of responder. The result revealed that with the increase of facial attractiveness and facial trustworthiness of proposers, people were more likely to receive the unfair allocations. Then a numerical example was given and the result further illustrated the rationality of model. Although there still exists some parts needed to improve, like the validation of actual decision results. But there is no denying that the current research established a systematic theory method for subsequent research and has good theoretical value and practical application value.

\section{References}

[1] Güth W, Kocher M G. More than thirty years of ultimatum bargaining experiments: Motives, variations, and a survey of the recent literature [J]. Journal of Economic Behavior \& Organization. Vol. 108 (2014) No. C, p. 396-409.

[2] Andreoni J, Petrie R. Beauty, gender and stereotypes: Evidence from laboratory experiments [J]. Journal of Economic Psychology. Vol. 29 (2008) No. 1, p. 73-93.

[3] Wout M V', Sanfey A G. Friend or foe: The effect of implicit trustworthiness judgments in social decision-making [J]. Cognition. Vol. 108 (2008) No. 3, p. 796-803.

[4] Fehr E, Schmidt K M. A Theory of Fairness, Competition, and Cooperation [J]. Quarterly Journal of Economics. Vol. 114 (2001) No. 5, p. 817--868.

[5] Oosterbeek H, Sloof R, Kuilen G V D. Cultural Differences in Ultimatum Game Experiments: Evidence from a Meta-Analysis ([C], EconWPA, 2004.) p.171-188.

[6] Pillutla M M, Murnighan J K. Unfairness, Anger, and Spite: Emotional Rejections of Ultimatum Offers [J]. Organizational Behavior \& Human Decision Processes. Vol. 68 (1996) No. 3, p. 208224.

[7] O'Doherty J, Winston J, Critchley H, et al. Beauty in a smile: the role of medial orbitofrontal cortex in facial attractiveness [J]. Neuropsychologia. Vol. 41 (2003) No. 2, p.147-55.

[8] Todorov A, Pakrashi M, Oosterhof N. Evaluating faces on trustworthiness after minimal time exposure [J]. Social Cognition. Vol. 27 (2009) p. 813-833.

[9] Yufei Jiang. An optimized the ultimatum game model [J].Vol.7 (2016) p.128. 